Article

\title{
Computational Thinking and Robotics: A Teaching Experience in Compulsory Secondary Education with Students with High Degree of Apathy and Demotivation
}

\author{
Belkis Díaz-Lauzurica ${ }^{+}$(D) and David Moreno-Salinas *, + (D) \\ Department of Computer Science and Automatic Control, UNED, C/Juan del Rosal 16, 28040 Madrid, Spain; \\ belkis.dialau@educa.jcyl.es \\ * Correspondence: dmoreno@dia.uned.es \\ † These authors contributed equally to this work.
}

Received: 26 July 2019; Accepted: 14 September 2019; Published: 18 September 2019

check for updates

\begin{abstract}
In present and future society, all individuals must be able to face the problems, risks, advantages and opportunities that will arrive with new paradigms in the labour market, social relations and technology. To reach this goal, a quality and inclusive education together with a proper and complete formation in technology (communications, robotics, programming, computational thinking (CT), etc.) must be imparted at all educational levels. Moreover, all individuals should have the same opportunities to develop their skills and knowledge, as stated in Goal 4 of the Sustainable Development Goals, Sustainable Education. Following this trend, in the present work, a practical experience about how to teach CT using robotics is developed, showing the results and evaluation of the lessons on robotics taught to students in their 4th year of compulsory secondary education, and where the students showed a high degree of apathy and demotivation. The teaching unit was based on an action research approach that includes a careful selection of pedagogical techniques and instruments to attract and keep the attention and interest of the students. In addition to the robotics lessons, a previous computational thinking training with Blockly Games was carried out, which contributed to noticeably increase the students motivation and to introduce them to the programming of robots. Moreover, gamification was used to motivate and evaluate the individual knowledge, and the students were required to present the work performed through a final project. The individual needs of the students were fulfilled with a daily monitoring. The results show that the pedagogical techniques, instruments and evaluation were adequate to increase the motivation of the students and to obtain a significant learning, showing how the teaching of CT may attract students that have lost interest and motivation, while providing them with abilities that will be essential for the learning throughout life.
\end{abstract}

Keywords: computational thinking; robotics; problem solving; educational games; competency-based education

\section{Introduction}

Training on technology, such as robotics, programming, computational thinking (CT), etc. from the first levels of education is essential for the construction of societies where the individuals are prepared for the challenges that will rise up with new paradigms in the labour market, social relations and sustainability [1]. Then, the transformation on the way of thinking, social values, and the actions required for a move towards sustainability and responsibility are of the utmost importance to construct a better society where all actors may be included. To reach this goal, a modification on the learning 
strategies in engineering and technology, and a transformation on the way of thinking on engineering and technology students is needed [2].

The above process involves all individuals during their student period, where they must receive a quality education and reach a formation that makes them responsible members of the society, aware of the risk and advantages of new technologies, where sustainability and responsibility play a central role. For this objective, students should receive an integral formation on technology and Computational Thinking (CT) that allows them to acquire the basic skills on math and technology and to face problems during their working and social life. Moreover, an special effort must be done to include all individuals, even those who are not interested in or pretend to abandon the studies, to avoid the interruption of their education, so that they all have the same opportunities. This is in line with Goal 4 of the Sustainable Development Goals (SDGs), Sustainable Education, for a quality education as the base to create a sustainable development [3]. In particular, Subgoal 4.1 is to "ensure that all girls and boys complete free, equitable and quality primary and secondary education leading to relevant and effective learning outcomes", Subgoal 4.3 is to "ensure equal access for all women and men to affordable and quality technical, vocational and tertiary education, including university", and Subgoal 4.4 is to "substantially increase the number of youth and adults who have relevant skills, including technical and vocational skills, for employment, decent jobs and entrepreneurship". Following this trend, in the paper at hand, a proposal for an inclusive education on $\mathrm{CT}$, programming and robotics is presented. The aim is to promote the interest of students with a high degree of apathy and demotivation, and with difficulties to continue with their studies. The underlying idea is to prevent these students from abandoning their academic life, and also to promote and develop their creativity, logical reasoning, problem-solving and project-design skills [4].

However, as programming may be a hard and complex matter, an adequate approach and methodology must be followed. In this sense, we can find several proposals for programming and CT teaching in a way that engages students with educational software or visual block-based programming environments [5,6]. For example, in [7], an analysis on the use of visual block-based programming for teaching introductory programming is presented using Scratch and App Inventor environments, and in [8], the combination of Lego Mindstorms and App Inventor is used for teaching novice programmers. In this line, in [9], a comparison between programming environments is carried out with the focus on secondary education. These works demonstrate how the students show more interest and ability in programming when this task is presented in an attractive way. Following this trend, in this study, a combination of Lego Mindstorm and online resources, such as Blockly Games and Kahoot, was used to teach programming and CT to students of 4th grade of secondary compulsory education.

Among the possible approaches that can be used to teach these concepts, project-based learning (PBL) approach stands out. PBL is at the core of the present proposal since it is a teaching and learning approach that is designed to attract students through the investigation and solving of authentic problems [10]. Moreover, the use of PBL in technology education makes the knowledge construction process explicit, and allows students to be aware of their own learning process [11], making it very appropriate for the teaching of Engineering and Technology [12]. The reader is referred to the work of Thomas [13] for a review on PBL projects. In this study, the PBL approach was supported partially with other approaches and methods to overcome all student profiles and learning styles, as described in Section 3.1.

Therefore, the scope of the present work is to show the applicability and give insight of a PBL based methodology to teach CT and robotics to students with disruptive profiles and to promote their motivation. Based on the qualitative research and the incidental sampling of the participants involved in the experience, the outcomes and results obtained may be transferred to similar or different contexts. This transference, or applicability of the results, on qualitative research is not a generalisation of the results for a wider population but to apply part of the results or their essence to other contexts, provide some guidelines to get a general idea of the problem studied and apply some solutions to other 
situations [14]. Then, the preliminary results obtained can provide guidelines for teachers that have to deal with similar situations. Moreover, the proposal can be adapted and modified depending on the different learning styles and profiles that the teacher may find, as well as on the previous experience with the students.

The contributions of the present work are fourfold: (i) to develop a methodology to teach robotics and Computational Thinking to students in compulsory secondary education; (ii) to show the application of the methodology and its results for a group of students with high level of apathy and demotivation, and analyse the advantages and drawbacks of the methodology proposed; (iii) to motivate the students with the use of tools to acquire the CT in a friendly and efficient way given their age; and (iv) to analyse the answer to the question "What are the personality profiles of top and low computational thinkers?" proposed in [15], given the real application of the methodology proposed. As far as the authors know, there are no studies about the teaching of robotics and CT dealing with similar student conditions.

The rest of the paper is organised as follows. In Section 2, an overview of the concept of CT and a summary of the education in $\mathrm{CT}$ and technology in different countries and regions is given. In Section 3, first, the theoretical foundations of the methodology imparted are presented, and then, the materials and methods used for the robotics sessions teaching are described. The results obtained and objectives fulfilled after applying the methodology proposed are presented and analysed in Section 4. Finally, in Section 5, a discussion about the results is detailed and some recommendations are given, and, in Section 6, the final conclusions are presented.

\section{Computational Thinking and Technology Education}

Computational Thinking (CT) was first defined in [16] as the thinking process that implies to solve problems, design systems and understand the human behaviour based on the fundamental concepts of computer science, and it has attracted a growing interest in the last decade. Multiple authors have incorporated new nuances or modified the concept of CT in the last years, such as [17-22], without reaching a real consensus about the cognitive abilities that define the training process of the CT.

In [23], the features that conform/construct the CT concept were summarised. These features are abstraction, as the ability to avoid unnecessary details; algorithmic reasoning, which could be defined as the necessary steps to reach a solution independently of the technology used; the ability to divide a complex problem into simple problems to make a clearer analysis; the abstraction automation, as a repetitive process to save time; debugging process, in which the results may be predicted and verified; and generalisation, as the way to extend the solutions obtained to new problems with similar characteristics.

In 2016, the Computer Science Teachers Association (CSTA) brought up to date the Standards in Computer Science for compulsory education based on the concepts and abilities of the CT [24]. Specifically, compulsory education is considered the base of the educative system, where the foundations of the integral education are established and the abilities to be used throughout life are developed. Therefore, the encouragement of collaboration, the work from a personal and social perspective, the healthy competition, and individual and group work, must be the essential means to give answers to the daily life situations and to prepare the individuals for their incorporation to the society and labour market. Following this trend, the teaching of the CT using robotics in a novel way during this life stage is of the utmost importance, and thus this education period has been chosen for the present study.

The teaching of CT in compulsory education, and particularly of robotics as one of the learning tools to acquire it, changes from one country to another, and it even changes from one region to another in the same country. Its inclusion in the curriculum for compulsory education advocates one of the two following approaches: to develop the ability to solve problems in daily life or to provide the adequate formation for future employments related to Information and Communication Technologies (ICT) and satisfy the demands of the labour market [23]. These two approaches are taken into account 
in the new educational programmes in countries of America, Asia and Europe, as can be seen in the following overview of the $\mathrm{CT}$ teaching in different educational programmes.

\subsection{Europe}

More than 20 European countries have incorporated CT into their educational curricula [25]. In $[23,26]$, the reader can find a detailed description about the way in which CT is included in the educational systems.

Among the European countries, Estonia stands out with its educational programme "ProgeTiger", where the teachers combine several resources (robotics platforms, applications from other disciplines, etc.) to provide an interdisciplinary educational provision. Similarly, Finland prepares the complete revision of its educational system with the training of teachers to teach the CT. In United Kingdom, the subject "Computing" incorporates the modelling of reality and the open reasoning, including different solutions to the same problem, together with the teaching of, at least, two programming languages.

In other countries, such as Germany or Spain, the CT teaching is done by regions. Germany stands out for the state investment in resources and teachers, and offers an optional subject for science students and courses that include practices outside the classroom environment. Regarding the educational system in Spain, different initiatives based on the optional subject "Tecnologia" taught in the 4th year of compulsory secondary education exist in each autonomous region. However, in some regions, such as Madrid, a specific subject has been created to teach the CT and/or robotics. Among the different initiatives in Spain, we can find Dr. Scratch [27], a tool to acquire CT at several levels (basic, intermediate, and advanced) and dimensions, being logic reasoning, data representation, user interaction, flow control, and abstraction among the most important dimensions. However, the Spanish Foundation for Science and Technology (FECYT) shows that a true integration of CT and/or robotics at a national level still does not exist, and it is also not applied in all educational institutions [28].

Other countries such as Austria, Cyprus, Lithuania, Hungary and Slovakia are immersed in the inclusion, in compulsory subjects, of CT related contents that were imparted in non compulsory subjects.

\subsection{The Americas}

A study about the design and implementation of educational robotics projects in Latin American and Caribbean countries was reported in [29], where their lack was remarked. However, there exist some projects with different importance and strength in each country: Project in Educational Technology or Educational Robotics in Mexico; Project Gira and its application Physical Etoy in Argentina; Foundation Omar Dengo in Costa Rica; and Project Colegio San Judas Tadeo in Dominican Republic, among many others. The main weaknesses of these projects and approaches are: they are mainly based on the development of guided constructions; a lack of standardisation of the teaching at a national level facilitating the improvement on programming abilities; and the lack of educational provision at higher education levels, as well as training programmes for teachers.

In North America, the United States proposed a virtual platform to enhance the CT teaching after some important institutions, such as Carnegie Mellon's Robotics Academy (CMU), the University of Pittsburgh's Learning Research and Development Center (PITT), Robomatter Inc., and the Robotics Education and Competition Foundation (RECF), concluded that, regardless of the many robotics groups in compulsory secondary education, they only worked on basic programming abilities that were oriented to solve the challenges proposed, with stand-alone solutions rather than generalisable and algorithmic ones [30]. In Canada, the integration of CT into the curriculum has been gradually envisaged since 2015. In the academic year 2016/2017, simple algorithms, visual representation of problems and data, the evolution of the programming languages and visual programming were 
included in lower secondary education, and it was subsequently extended to upper secondary education [23].

\subsection{Asia}

South Korea, China, Singapore and Japan have announced the integration of CT into compulsory education. In Singapore, programming is a compulsory subject and robotics is taught from the first years of education. This is in line with its robotics national programme, which will promote in 2020 the construction of robots for multiple productive areas [31]. Japan stands out by its great production of new technologies, robotics research, and the high level of industrial application of innovations. All the above have an important effect in students' formation, making Japan one of the countries with the highest level of ICT integration in classrooms [32]. Moreover, robotics and drone assembly are taught in compulsory secondary education [33], and programming will be compulsory in 2021 [23]. China will include robotics in the national educational curriculum from the early years in compulsory education [34], while South Korea has the programme "Software Education" in pilot phase, which is compulsory at all education levels and includes also the teaching of drone assembly [35].

\section{Materials and Methods}

In this section, the theoretical foundations of the methodology used to give lessons of programming and robotics are firstly explained together with the expected goals to reach by applying the described methodology. The group of students and its characteristics, the different instruments and tools used for the application of the methodology and the data collection used to evaluate the results are presented next.

\subsection{Methodology: Theoretical Foundations}

The methodology proposed in this work is based on the conjunction of planned activities for each learning style, different forms of evaluation and the combination of resources that allow to deal with students with a high degree of apathy and demotivation. The foundations of the proposal reside on the Project Based Learning and constructivism so that the students are aware of their learning process, overcome real problems, acquire the tools and knowledge to solve different problems and situations, and continue their learning throughout life. Therefore, the core of the teaching and learning process is based on the investigation and solving of real problems and on the active learning of the students by their own experience. This approach is appropriate for programming and robotics sessions since the students are aware of their learning process with an explicit knowledge construction $[10,11,36]$.

In addition, part of the present study is based on the research shown in [15], where an analysis of the non-cognitive and cognitive abilities that may be developed by CT teaching is carried out. In the conclusions of this study, the personality profile of high and low level computational thinkers is outlined. It is remarked how the profiles of the computational thinkers have changed in favour of extroverted people, how the own definition of extrovert/introvert is being changed, how some students that showed initially disruptive attitude had a very well adaptation to programming experiences and also how in many situations the student abilities and performance are improved with the new methods and ways of communication. This point of view, given by a general statistic, can be tested and/or validated from the educational practice perspective proposed and carried out in the work at hand.

The practice experience is based on the initial design of a competence-based teaching unit. In order for the students to acquire the different competences, general aspects were described in the teaching unit: reasons that motivated the design of activities given the different learning styles, planning of the sessions from the simplest concepts to the most complex concepts, active role of the students during their learning, collaborative work and collaborative projects to solve the different challenges, support resources to improve the teaching of robotics lessons, treatment of the didactic error, conception of evaluation, etc.

The other concepts and approaches that served as a base to construct the present proposal were: 
- Inductive methods and qualitative research [37], to focus the proposal on the teaching-learning process rather than on the results. Among the different methods of the qualitative research (ethnographic research, case study, discussion group and evaluative research), the action research approach was chosen due to its own features: (i) The continuous implementation of actions and decision making over the educational practice to improve the quality of the learning-teaching process was carried out in each session. (ii) The implementation of a circular process based on planning, action, observation and replanning was applied during the planning of the teaching unit and its modifications, which were conditioned by the evolution of the sessions. (iii) Among the different approaches of the action research (of the teacher, participative and cooperative), the teacher approach was selected because the methodology was focused on the teacher experience, being crucial the understanding of the context, systematisation, analysis and study of the reality [38].

- The constructivistic approach adopted in this work, which promotes the effort and active participation of the student during the learning, was based on cognitive, conductual and social theories proposed by different authors. Among them, the contribution of Piaget to the constructivism stands out, which describes the assimilation and accommodation mechanisms by means of which an individual is able to incorporate new information and knowledge to its previous knowledge [39].

- The socio-constructivistic theory of Vygotsky, which explains the importance of the close development area (CDA) for learning and places the learning process as dependent of the individual activity [40], substantiated this proposal where the students were growing in autonomy while the teacher served as a guide.

- Other cognitive theories, such as the theories of Ausubel [41], and Papert [42], give great importance to the significant learning as the way to really acquire knowledge. Specifically, Papert defended the "hard fun" or activities that imply an additional motivated effort by the students to obtain a product or result. In addition, in the Theory of Learning by Discovery, Bruner [43] related the knowledge to the cultural context and defended that the proposal of problems with increasing complexity is essential for the intellectual development. These aspects were taken into account for the planning of activities, either for introductory or programming and construction challenges, or for support games. See the "tram" project in Section 4.2, in which the students solved several design, programming and construction situations.

- Additionally, planning the challenges during the sessions according to the student abilities to maintain a high level of motivation was based on the flow state theory of Csikszentmihalyi, who suggested that work is preferred to entertainment and that knowing how to manage the objectives may drive to a state close to satisfaction or happiness [44].

- Some of Thorndike's laws that rule the learning were revealing and helped to construct this proposal. This was the case of the transference law, whose basis was useful to plan the application of different learnings in different situations; the novelty law, which justifies the incorporation of different robots and situations as examples; the exercise law, which was used to practice the new knowledge and strengthen it; and the extinction law, repeating some concepts and practices so as not to forget the knowledge acquired [45].

- Finally, the encouragement of innovation and creativity in the challenges proposed depending on the learning styles was based on the published works of Robinson, who stands up the importance of learning from risks and errors, and who states that creativity can be learnt and that the creative education must address all students to strengthen their individual abilities [46].

Therefore, taking into account the theoretical foundation commented, and by knowing the present education trends in the different countries, the present work attempts to answer some of the European innovation and research programme H2020 guidelines, particularly the one referred to "Reflexive, innovative and inclusive societies", and Goal 4 of the Sustainable Development Goals (SDGs), Sustainable Education, for a quality education for all individuals as the base to create 
a sustainable development [3], while dealing with students of compulsory secondary education with high demotivation, which are on the eve of a new social and working life.

Therefore, the main goals to be achieved, and considering the group of students, were:

- $\quad$ Promote the interest and motivation of the students.

- Make the students aware of their own learning and an active part of the knowledge construction process.

- Improve the behaviour and the academic grades.

- Develop programming and CT skills and interest about math and technology education.

\subsection{Work Group}

The group was composed of eight students with some particular characteristics:

- Five of them had repeated a previous grade of compulsory secondary education and two of them had repeated two grades. One of these two latter students had many difficulties for abstract reasoning.

- $\quad$ Some of them came from other educational institutions due to behavioural problems.

- In general, they had very little interest in the different subjects, as their poor grades and their daily behaviour showed. They also did not show significant learning since it was very difficult for them to incorporate new knowledge, and also to transfer it to other areas of life and studies.

- Three of them were also students with special educational needs (SSEN) with curricular adaptations, two students with adaptations corresponding to the 4th and 5th years of compulsory primary education and the other one with adaptations of the 2nd and 3rd years of compulsory secondary education. For these students, the activities were adapted to their learning styles and their capacities by recommendation of the educational guidance counsellor of the institution.

Notice that the sampling was incidental instead of probabilistic, with a very homogeneous group. None of the students had previous experience or knowledge on robotics.

The evolution and development of activities, of the evaluation, as well as the most appropriate working methodology for the students were taken into account to evaluate the work carried out by them and the progress of the robotics sessions. A total of 30 sessions were programmed for this group.

\subsection{Instruments}

Different instruments were used for the lessons:

- Interviews and initial tests were developed to know the different profiles, learning styles, knowledge and possible cognitive deficiencies of the students. Initially, open interviews were carried out to ask broad questions, and subsequently, semi-structured interviews were developed.

- $\quad$ Mindstorm kit (EV3), with programming software included, was used for the experimental classes. In [47], a study of the possibilities of using Lego Mindstorm for teaching can be found, as well as the importance and versatility that the modularity of the kit provides. It allows physically modifying the robots with assembling pieces and to modify their behaviour based on algorithms specified by visual programming or text based programming.

- The following web resources were utilized: (a) Blockly Games, which is an online didactic game for initiation in programming. The game consists in solving challenges by ordering programming structures presented as blocks. The game has seven activities, with ten challenges each, in increasing difficulty [48]. This game was used as support for the robotics lessons. (b) Kahoot, which is a game oriented evaluation tool, whose advantages are described in [49].

\subsection{Data Collection and Analysis}

The classes followed the planning proposed in the teaching unit for $30 \mathrm{~h}$ and the analysis of the data collected was based on the qualitative analysis as stated in Section 3.1. 
The daily activities and their development were recorded in a class diary by the students and by the teacher, and observation was also used as data collection instrument. The observation of students was systematically planned and controlled in relation to the overall objectives and the content of the lessons. It was subject to verification and to validity and reliability control in relation with the results observed from the different activities [50].

The data collected were also studied using a data analysis program (Atlas.ti [51]) to improve the quality of the educative research, since it provides more coherence and rigour to the analytical procedures [52]. This software allows the data segmentation in units of meaning, their codification and to relate concepts and categories. The election of this program is due to its ease of use; the capacity of working with different data types; the possibility of ordering, combining and filtering the codes in a search, and of generating maps and relations, hypotheses and theories; etc. All these features give a significant rigour to the program outcomes [53].

The aspects analysed and evaluated using the qualitative methodology were:

- Programming of the robot: The student activity was analysed during each programming session to evaluate if every step was correctly taken. The different solutions proposed were also considered for the evaluation, as well as their suitability and effectiveness. The diary development and attitude were recorded in the teacher diary.

- Behaviour during the project: The behaviour of the students was a critical factor in the methodology since the group was highly disruptive and was very demotivated at the beginning of the sessions. Their behaviour changed during the experience from passive and uninterested to active and interested. The behaviour was recorded in the teacher diary every day.

- Use of Blockly Games and its suitability for the robotics lessons: The use of Blockly Games as a complementary tool was very important to introduce, in a ludic way, programming concepts and make all the students be part of the learning process. The data collection was carried out by analysing the results and solutions proposed by the students to each challenge.

- Kahoot exam: The questions made for Kahoot exam were representative of each of the contents imparted in the sessions and of the skills to be developed by the students. Thus, each concept imparted in the teaching unit could be evaluated and compared with the results obtained in the rest of activities.

- Evaluation results: Exam results and presentation of the project, which showed the good performance of the group.

The rigour on the qualitative methodology application and triangulation methods to analyse the results was secured by different factors:

1. Dependency: The data must be revised by different researchers and they have to reach coherent interpretations [54]. To guarantee the dependency:

- The events were classified in the diary with symbols, indicating date, time, goals to reach and competences to work. This was useful to assess the quality of the data during all the sessions.

- The direct observation was done in condensed and detailed form, justifying each action and its causes, with an easily comprehensible language, so that other researchers can recover and replicate the information. For each activity, the logged data were: activity, date, time, worked competences, resources, students development, comments, behaviour, decisions and changes made during the activity.

- Several interviews were carried out with the institution's counsellor and teachers.

- The observations were contrasted with another teacher that was in the classroom and also with external researchers.

- A qualitative analysis program (Atlas.ti) was used to standardise the course of actions, the collaborative research, and the generation of hypotheses, graphs, etc. 
- The data matching was demonstrated by: (i) the acquired skills with Blockly Games and the demonstrated skills during the robot programming; and (ii) contrasting the exam results in Kahoot with the empirical evidences analysis with Atlas.ti.

- Decisions and agreements with the institution observers based on the bimonthly assessments, taking into account the participants attitude and the goals reached.

2. Credibility: To ensure that the researcher has the complete meaning of the participants experiences, particularly those related with the problem statement [54]. The credibility was improved by:

- long stays in the classroom by the same teachers, which allowed to broaden the thinking and evaluate the changes;

- applying this same experience with other students with different characteristics;

- collecting quantitative data from qualitative data; and

- competence evaluation based on projects, taking into account the specific characteristics of the students.

3. Confirmation: To demonstrate how the bias of the researcher has been minimised [55]. In addition to the above triangulation methods, during the activities with examples and exercises, the same solving method was applied to different exercises and different solving methods were applied to the same exercise.

4. Transference: To apply and transfer the results (or part of them) to other contexts and avoid inconsistencies in the research findings [56]. For this reason, findings of the present work were transferred to another context (in this case a group very motivated and with good grades):

- The same teacher gave the robotics lessons, keeping the same methodology to promote the motivation.

- The same concepts on programming were taught following the same didactic unit.

- The knowledge on programming and robotics was similar on both groups before the lessons.

\section{Results}

In this section, the main results obtained are detailed. The results are described separately for each of the actions carried out during the sessions. The discussion and interpretation of the results is done in Section 5.

\subsection{Robot Programming}

This was the activity that consumed more time, since this was the main objective of the proposal. Based on the previous teaching experience with the students and on their lack of experience and knowledge on the subject, the different examples and concepts were presented in a simple way to make them more complex in subsequent sessions. This allowed us to prevent the students from feeling that they were working too long on the same example/concept, and to complete the exercises correctly and on time. In addition, the introduction of new elements in the explanations and the use of multiple examples to explain the concepts helped to increase the attention of the students.

The work with the Mindstorm software was only oriented to learn the programming structures needed for the project. This decision was correct since the students were able to apply the right structures in different situations, they understood the use of these structures and they were able to introduce modifications.

During the sessions, the most frequent difficulties that the students met in the programming of the robot were (presented from lower to higher difficulty):

- Code reading: From Session 8, the students began to adequately understand the code and they were able to solve simple code problems. They showed a qualitative progress from Sessions 14/15, in which they correctly understood all components and elements of a program.

- Loop concept: Some theatrical explanations about problems and the use of pseudocode were employed to explain loops, which were well received by the students. 
- New functionalities integration: As of Session 12, the students showed difficulties to introduce new working conditions on the code by keeping the previous requirements. In this phase, the students required the help of the teacher: they knew what they had to do and what part of the code they needed to work on, but they did not realize "how".

- Variables: The concept of variable was very difficult for them to understand. Explanations on blackboard helped them to understand the concept, but a relevant result on this aspect was not possible.

- Fault diagnosis: After 19 weeks, the students were able to diagnose programming errors by themselves. The execution errors diagnose required a longer time to be solved.

The general order in which the developed skills were obtained during the programming sessions is shown in Table 1, in addition to the level acquired. This level was catalogued as high, medium or low depending on the abilities and skills acquired and demonstrated:

- High: The student shows sufficiency and autonomy.

- Medium: The student works well, but still needs some help in specific moments.

- Low: The student does not show (or shows few) abilities or knowledge.

Table 1. Developed skills by the students.

\begin{tabular}{ccc}
\hline & Developed Skills & Level Acquired: H (High), M (Medium), L (Low) \\
\hline 1 & Know the sensor functionality & $\mathrm{H}$ \\
2 & Load the program on the processing unit & $\mathrm{H}$ \\
3 & Use of the programming environment & $\mathrm{H}$ \\
4 & Understand pseudocode & $\mathrm{H}$ \\
5 & Identify and read program sections & $\mathrm{H}$ \\
6 & Understand loops and their functionality & $\mathrm{H}$ \\
7 & Identify programming structures from the statement & $\mathrm{M}$ \\
8 & Link conditions and functions & $\mathrm{H}$ \\
9 & Detect and identify problems & $\mathrm{M}$ \\
10 & Diagnose and solve problems & $\mathrm{M} / \mathrm{L}$ \\
11 & Understand condition variables & $\mathrm{M}$ \\
12 & Work with variables & $\mathrm{L}$ \\
\hline
\end{tabular}

Notice from the developed skills shown in Table 1 how the students acquired most of the skills with a high level. Most of the time they were conscious of their own progress, which helped them to participate more and feel that they were learning. In most of the sessions, they showed a very good behaviour and they were interested in the concepts taught. It is important to notice that this good attitude was totally opposite to the one showed in the previous semester and even in the previous years, where they did not show interest in the academic studies.

Regardless of the good results in most of the skills, they did not show a significant evolution in the work with variables. This concept was difficult for them and together with the limited time in which this experience was applied and the cognitive deficiencies for abstract reasoning showed by the students from the first session, it was not possible to achieve a good result.

However, in general, the students showed a well behaviour and a significant interest in the sessions, in contrast to the behaviour and interest showed during the rest of the academic year. This made it clear that, with the adequate programming and learning methods, a group of demotivated and conflictive students may recover the interest and the good academic results.

\subsection{Tram Project: Robot Construction}

The robot to construct was composed of two parts: a tractor wagon and its corresponding trailer, so that it had a similar functionality to a tram. It had to follow a given path, stop after pressing a button and deploy a ladder for the passengers, as well as to recognise the traffic lights and their commands. In addition, the trailer had to keep the front wheels in the correct direction to turn, following the wagon trajectory, so that a coupling system between wagon and trailer had to be designed too. 
An initial prototype was constructed following the test-error approach for the following reasons:

- In a practical way, the students were verifying the feasibility of using the different kinds of pieces.

- They could notice in a fast manner that the robot must be constructed step by step to fulfil all the project requirements. In addition, in this initial phase, they did not have to worry about the design so they did not have any pressure to obtain the best result possible. According to the final result obtained (the tram), the students were able to construct a prototype that could solve most of the requirements stated during the conception of the robot (Figure 1).

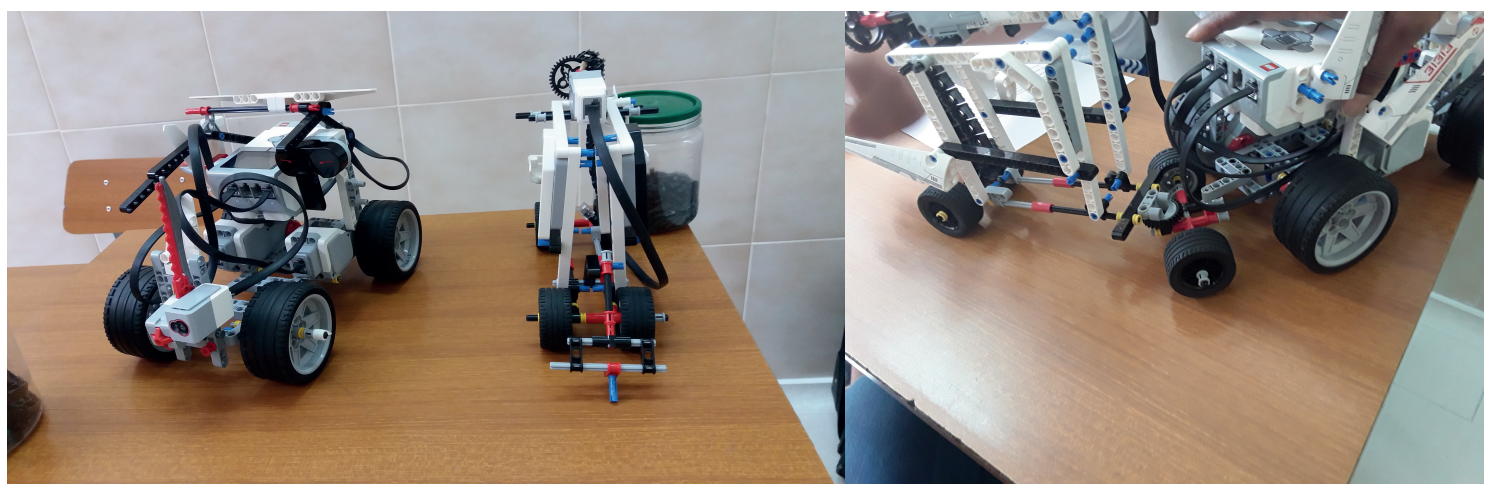

Figure 1. (left) The first, non-functional version of the tram; and (right) improvement on the construction and functionality.

During the programming of the robot, the students acquired the elemental knowledge and concepts as they were able to introduce most of the changes and modifications in the correct place, and they even identified and located the errors.

The additional objective of working in groups, so that the students collaborated and helped each other during the documentation of the project, was not completely achieved since some of them simply took care of their own tasks, which depended on the previously assigned roles in the groups.

An examining board was created for the project and documentation exposition so that the students had to explain the different features of the project and the difficulties encountered along the work. By doing so, the students had to prepare a formal presentation and develop the linguistic competence.

However, they showed apathy in this activity, since this kind of "classic" activities led them to a state of apathy similar to what they showed before the project. It became evident that all activities out of the scope of their learning style were meaningless for them, so that, from a pedagogical point of view, this kind of activities was not adequate for them.

To summarise, the developed skills by the students during the project are shown in Table 2, in addition to the level acquired. This level was catalogued as high, medium or low depending on the abilities and skills acquired and demonstrated:

- $\quad$ High: The student shows sufficiency and autonomy.

- Medium: The student works well, but still needs some help in specific moments.

- Low: The student does not show (or shows few) abilities or knowledge.

Table 2. Developed skills by the students.

\begin{tabular}{ccc}
\hline Order & Developed Skills & Level Acquired: H (High), M (Medium), L (Low) \\
\hline 1 & Work and understand the examples & $\mathrm{H}$ \\
2 & Think on different solutions to a problem & $\mathrm{M}$ \\
3 & Predict problems and solve them & $\mathrm{H}$ \\
4 & Conceive an idea from the draft until its execution & $\mathrm{H}$ \\
5 & Flexibility on the design & $\mathrm{H}$ \\
6 & Teamwork & $\mathrm{L}$ \\
\hline
\end{tabular}


The correct execution of the robot ( $40 \%$ of the final qualification), the oral presentation ( $20 \%$ of the final qualification), the fulfilment of requirements ( $30 \%$ of the final qualification) and the documentation prepared ( $10 \%$ of the final qualification) were considered for the final grade.

Therefore, from this activity, we can conclude that the work with a physical robot motivated them to try different solutions and to test the development of the robot. They were committed during the entire construction and testing process, showing that project based learning was better adapted to their capacities and motivated them. However, they showed again a disruptive behaviour when they were requested to work on activities that were not of their interest, such as the documentation and presentation of the project. These latter activities must be worked on too, so that the students may developed social and linguistic skills, but the classical approach followed on these two activities showed that, even for these typical activities, a different approach must be applied. The study and analysis of these different and new approaches deserves a deeper study, to adequate these activities to the different profiles and learning styles.

To summarise, the results obtained in the robot construction and programming, and in the detection and resolution of errors, showed that the students reached a significant knowledge in relatively few time. They also showed that highly apathetic and demotivated students can regain the interest and motivation by adapting the activities to their learning styles, with the combination of theoretical and practical examples based on robotics and CT.

\subsection{Blockly Games Challenges}

The use of the Blockly Games challenges in some of the sessions was of great help to solve some questions:

- It helped to train the resolution of problems that required the use of programming structures, which were later used with Mindstorm.

- These challenges required less effort from the teacher once the students learned the work dynamic. After the third/fourth challenge, the students started to work autonomously.

- This format was adequate to work with classes with a larger number of students, and allowed alternating with other activities that required more implication by the teacher.

- It helped to motivate the students: as they were progressing with the challenges, they were more motivated to work with the robot. Moreover, students continued working on this activity one week more than planned on their own.

Therefore, this kind of activity helped to prepare the students to work with the robot programming, trained them in logic, and improved their concentration and individual work. The development of the Blockly Games challenges was also considered for the final evaluation of the students.

This activity was very welcomed by the students, as they took it as a game while they were learning the different programming concepts. Moreover, this kind of activities allowed the teacher to organise the classroom to attend special needs from some students. In this sense, if some of the students needed more help for the construction of the robot or the robot programming, the rest of the class may be working with Blockly Games in the advanced challenges. Doing this way, every student can advance at their own pace and they do not feel the pressure of the rest of the students.

\subsection{Daily Evaluation}

The results show that, although sometimes the mood or behaviour was not the best, the students progressed in the programming and development of applications and constructions. The improvement on their behaviour during the experience was very significant and the institution showed interest in continuing with the methodology for similar cases of apathy and demotivation.

\subsection{Kahoot Evaluation}

The results show a qualitative improvement in the student progress. It is important to remark the high concentration during the exam in spite of the lack of knowledge on some questions. 
This contrasted with their attitude in the initial test, where they left unsolved some questions and they did not show interest. The students acquired good command and knowledge of the programming structures, which were unknown for them at the beginning of sessions. Besides, the understanding of the pseudocode was positively evaluated. The results obtained in the Kahoot exam were equivalent to the evaluation made throughout the programming sessions and the robot constructions, showing the adequate data collection and analysis in the different evaluation methods.

\subsection{Qualitative Analysis}

As indicated in Section 3, the daily activities and their development were recorded in a class diary by the students and by the teacher, using direct observation as data collection instrument. This direct observation was subject to verification and to validity and reliability control in relation with the results observed from the different activities [50].

The data collected were also studied using the data analysis program Atlas.ti [51]. The results provided show how the Blockly Games challenges and the programming sessions were the more motivating activities (see Figure 2). Programming in pseudocode was less engaging, even though they understood the programming concepts very quickly with this activity. Although the motivation was not always high, the qualitative change was evident. This confirms the hypothesis that the interest and motivation of the students would increase with this kind of activities.

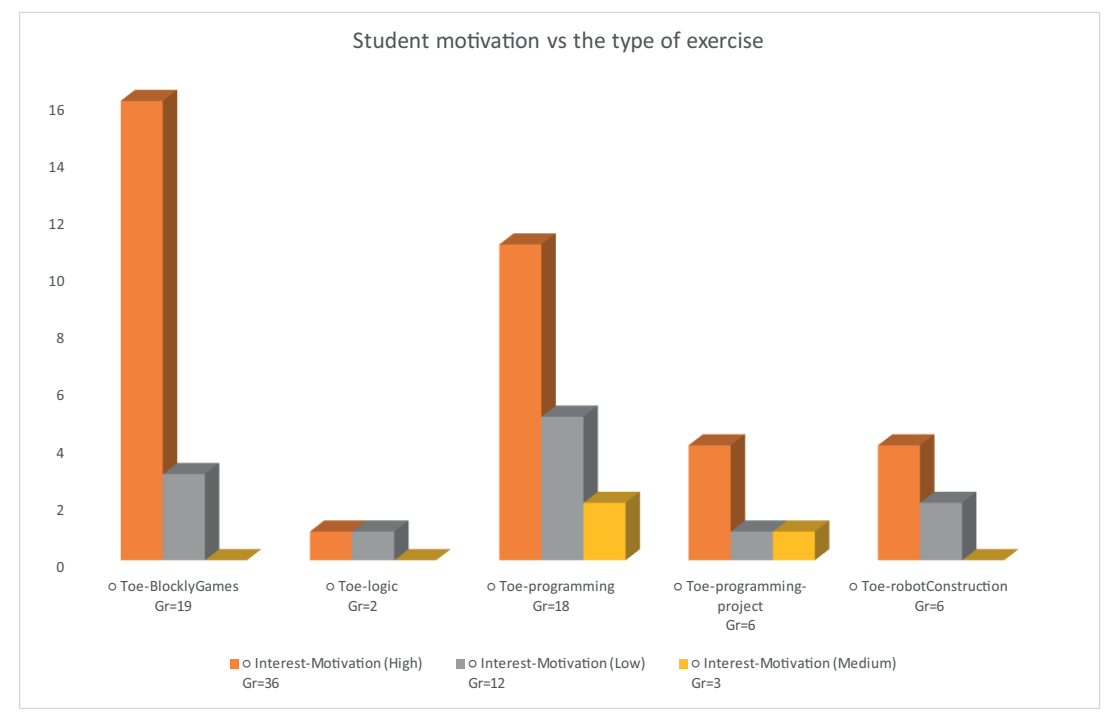

Figure 2. Students motivation for the different types of exercises (toe). Gr is the number of times that the corresponding concept is referenced in the empirical evidence.

Similarly, complex cross-studies were developed to test if the exercise resolution time decreased as the students solved more exercises and learned new solving methods. This was found taking into account the resolution time of similar exercises with different solving methods and the resolution time of different exercises with already known solving methods. Given the empirical evidence, the results show that the resolution time is low-medium to solve similar exercises with new and different methods and medium-high to solve different exercises with known methods. Therefore, for these students, the response time tends to decline for the resolution of similar exercises.

The reasoning of the students for the different activities improved significantly (see Figure 3). It can be seen how the reasoning in the Blockly Games challenges was medium-high, and in the programming sessions previous to the tram project it was medium. Afterwards, during the tram project and for the resolution of similar exercises with different solving methods, the autonomy and reasoning of the students grow in great manner, confirming the hypothesis that the reasoning and the ability to solve new questions is improved after the planned activities. 


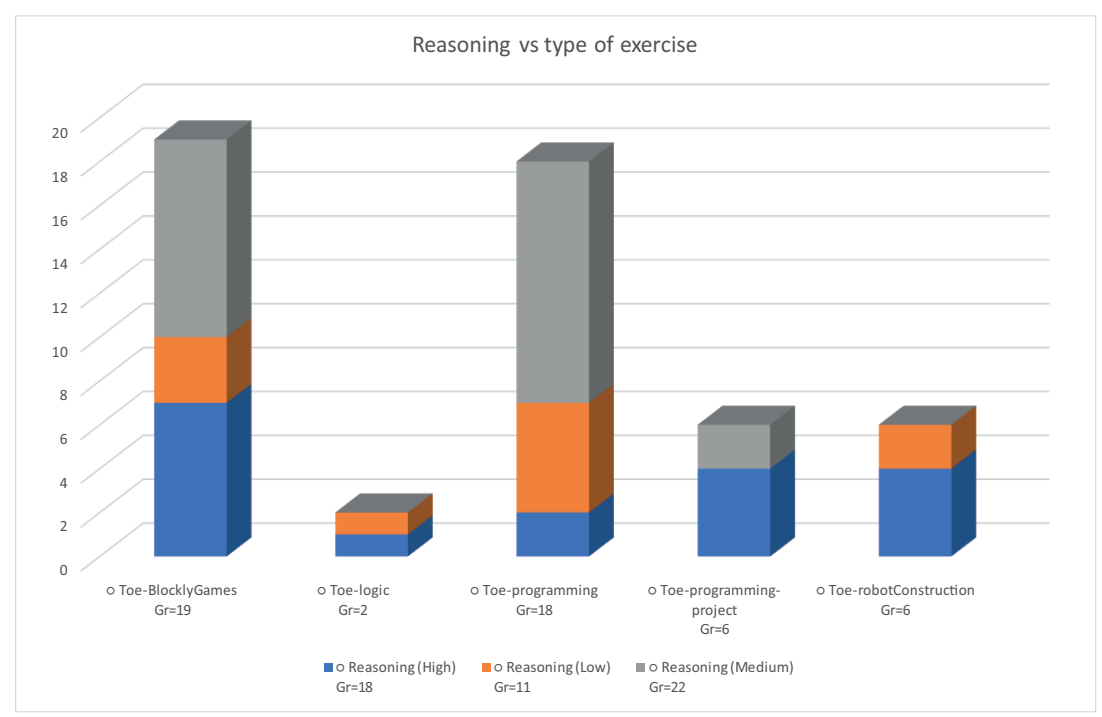

Figure 3. Students reasoning for the different types of exercises (toe). Gr is the number of times that the corresponding concept is referenced in the empirical evidence.

\subsection{Objectives Fulfilment}

According to the obtained global results:

1. The resolution time of similar problems decreased, i.e., the reasoning and the ability to solve new questions improved.

2. As the students spent more time with Mindstorm, their ability to acquire the programming structures logic improved, i.e., the students used the knowledge obtained from past experiences.

3. The academic grades and the interest for the studies increased.

4. The motivation and compromise increased: at the end of the sessions, the students continued working with Blocky Games for one more week on their own.

5. Regarding the results of the programming exam previous to this proposal, the improvement in the understanding and work on programming structures was very significant. This fact is also evident in the autonomy that the students showed during the tram project.

6. The behaviour of the students improved significantly, keeping the attention and interest in most of the sessions.

The guarantee of the results is underpinned by the different triangulation methods undertaken along the research at different levels:

- Several methods of evaluation ensured that the knowledge acquired was significant: construction of robot, robot programming, programming challenges, teacher diary, and final exam.

- Different methods were used to make the same program: in pseudocode, in the project and in the previous programming sessions.

- The application of a given method was used to solve different problems in robotics.

- The views and opinions of different agents and sources in education were constrasted: researchers, teachers of the institution, institution counsellor, the diary, the initial diagnosis test, etc.

- The results were observed from different points of view, including the behaviour of the students and the learning diary.

- Several resources were used to check the CT acquisition by the students: Blockly Games, Mindstorm, pseudocode, exams, etc.

In addition, some limitations were found during the application of the present proposal, which had an impact on the analysis undertaken:

- The proposal was implemented during the last quarter of the academic year, thus the follow up of the students was limited. 
- A limited time window to implement the proposal did not allow the application of other ideas included in the teaching unit.

- At the end of the sessions, the students did not reach enough abstraction level to solve high complexity problems, thus the results to this kind of questions were lower. This limitation may be caused by the cognitive deficiencies on abstraction that the group showed at the beginning of this proposal.

Regardless of the above points, in general, the goals considered in this proposal were achieved. Note: It is important to remark that all students passed the evaluation.

\subsection{Results Transfer}

The results transfer was done over a group of students of 3rd year of compulsory secondary education. The group was composed of nine students with high motivation and good grades, with some students with particular characteristics:

- A student with special needs and reduced syllabus.

- A student with ADHD (Attention-Deficit/Hyperactivity Disorder).

- A student with high intellectual capacities.

The programming sessions were combined also with the Blockly Games challenges. The order in which the developed skills were obtained during the programming sessions is shown in Table 3. The level was catalogued as high, medium or low depending on the abilities and skills acquired and demonstrated:

- High: The student shows sufficiency and autonomy.

- Medium: The student works well, but still needs some help in specific moments.

- Low: The student does not show (or shows few) abilities or knowledge.

Table 3. Developed skills by the students.

\begin{tabular}{ccc}
\hline Order & Developed Skills & Level Acquired: H (High), M (Medium), L (Low) \\
\hline 1 & Know the sensor functionality & $\mathrm{H}$ \\
2 & Load the program on the processing unit & $\mathrm{H}$ \\
3 & Use of the programming environment & $\mathrm{H}$ \\
4 & Understand pseudocode & $\mathrm{H}$ \\
5 & Identify and read program sections & $\mathrm{H}$ \\
6 & Understand loops and their functionality & $\mathrm{H}$ \\
7 & Identify programming structures from the statement & $\mathrm{H}$ \\
8 & Link conditions and functions & $\mathrm{H}$ \\
9 & Detect and identify problems & $\mathrm{H}$ \\
10 & Understand condition variables & $\mathrm{H}$ \\
11 & Work with variables & $\mathrm{M}$ \\
\hline
\end{tabular}

The experience was considered very successful: all students were very involved and motivated in the sessions, showing a good attitude and behaviour, obtaining good and high grades. Moreover, three of them completed the work with variables. It is important to remark that, in this case, the group was committed and dedicated.

\section{Discussion}

The starting point of this work was a group of apathetic and not interested students, with important curricula deficits and with some adaptations to previous courses. Moreover, some of them had repeated some courses and also had behavioural problems in other institutions.

For redressing this situation, the present proposal was designed: A programming and robotics course inspired in the PBL methodology, where additional features were used to keep the attention and interest of the students, such as gamification (Kahoot and Blockly Games) or work group with roles. The learning styles of the students were taken into account to keep the attention, so that the 
sessions may be easily modified according to the student behaviour and their state of concentration. Doing this way, the students were involved in the learning process during the experience, both in the programming sessions and in the robot project sessions.

The results described in Section 4 show, in general, a good proficiency of the group in the programming skills taught along the proposal. They were interested in most of the planned activities: they worked actively in the robot tram project, they were very interested in the Blockly Games challenges, and they participated in the exam with good attitude. Moreover, the analysis of the time that each student requires to solve a task in Blockly Games, before and after the robotics sessions, can be an indicator of the acquisition of CT skills, as reported in [57]. In addition, similarly to the results reported in $[8,9]$, the students showed interest in increasing their knowledge and enjoyed the different tasks and tools used, being visual block-based programming environments an effective way for programming learning. Thus, with the adequate environments and tools, the improvement on programming and CT skills may be very significant and may attract more students to technical studies. In a general way, the steps planned in the methodology were a success and an appropriate guide for the teaching of students with very different characteristics, showing its applicability, and the possibility for application to similar contexts and for transfer to different contexts. This was demonstrated with the application of the same methodology to a very different group that showed high motivation and good grades. The outcomes obtained from the new application of the methodology were similar to those found with the group of study, showing the students a high commitment and interest in the activities and actively participating in their own learning.

The most problematic sessions were those in which variables were explained. It is important to notice that some of the students had specific difficulties in abstract reasoning, as well as a general indifference to the education process. However, this fact did not discourage them, and, despite the low results obtained regarding this concept, they did not lose their commitment and interest.

It is important to notice that the results reported in this work are preliminary results. Thus, some inherent drawbacks have to be taken into account for an objective evaluation of the results achieved: It was applied only during the last quarter of the academic year and the group of students was relatively small. On the one hand, it should be applied during a longer period of time to evaluate if the increase of interest and commitment by the students do not decrease, and, if necessary, to study the possible adaptations in the proposal to avoid it and keep the interest. On the other hand, the results were obtained over a limited group of students, and, although they are promising and some general conclusion can be obtained from a small group of participants [58], further analysis should be carried out with a larger number of participants. In addition, the small group considered helped to deal with the students in a very close way, detecting negative situations (behavioural problems, lack of attention, distractions, etc.) very quickly and allowing to act over them. For a larger group of students, a single teacher may have problems adequately meeting the needs of all students and correctly evaluating their progress. In this case, the institution should consider a support teacher for the classroom.

In addition, this kind of methodology may be applied for practically any institution of any country, since the investment needed to carry out the proposal is very small. The cost of the Lego Mindstorms kits is not high, and also their use can be combined with the Blocky Games challenges if there are not enough kits. Moreover, the programming and robotics sessions may be complemented with simulation environments that help to understand the concepts and also to carry out projects of higher complexity whose cost could be too high to acquire the required components. In this line, there are important efforts by the researchers to develop simulation environments for programming learning and to study their efficacy $[8,9]$.

As regards the conclusions in [15] with respect to the question "What are the personality profiles of top and low computational thinkers?":

- We agree that external digital world sways in the internal psychological world, acting as the modern zone of proximal development (ZPD) of Vygotsky. In addition, with the development 
of new ways of communication, the individuals are looking for the interaction with the world that best fit their personalities. However, for this reason, the present concept of introversion is very fuzzy, thus the concept should be redefined as well as the abilities that encourage the different individuals.

- Taking into account that the student profiles on this experience tended to introversion with disruptive attitudes, and that a qualitative improvement in the computational thinking and programming skills was clear in the evaluated aspects, we can state that the use of motivating tools in combination with an adequate pedagogy can make that these problematic profiles can start to find a new space (programming field) where they can develop their abilities and find a new opportunity to continue their studies with success.

Therefore, this proposal may be used as an example that this change in the profiles of the good computational thinkers from introverted to extroverted students, as indicated in [15], may be caused by the progressive modification of the methodologies and the learning methods more than a question of introversion/extroversion of the students. Then, these teaching and learning methods would be better adapted to some students personalities, interactions and ways of learning according to the new technologies, but that under the appropriate orientation and methodology any profile could find motivation and interest in learning and obtain good results along their academic life.

\subsection{Robotics Sessions Prospective and Recommendations}

The positive results obtained motivated the institution to buy additional Mindstorm kits to continue with the methodology proposed in the subsequent academic years, showing the importance and novelty of the results obtained with this group of students and the applicability of the methodology and teaching unit.

For its application during a longer period of time, it is convenient to include additional tools, such as simulation environments, interactive programming tools and different kits, such as Arduino, App Inventor, Scratch, etc. This would help to make a dynamic proposal, in which different tools may be combined and used together to teach the different concepts while keeping the interest and attention of the students. Moreover, these tools may be used to develop the skills of the students over their academic years, keeping and using tools well known for them.

For the present proposal, project-based learning, complemented with other methodologies, was considered for the teaching unit and the robotics sessions. For a longer course, the use and combination of different methodologies for different units, such as flipped classroom, gamification, etc., might also be a good initiative for making the sessions more attractive and to try to deal with all the learning styles. Thus, the wide range of possibilities and approaches makes the education in technology a very active and vibrant field that requires the biggest efforts from our side.

\section{Conclusions}

Goal 4 of the Sustainable Development Goals is centred on quality education. This goal is focused on the access to an education of quality for all students, in which they can develop their skills and acquire the knowledge for their learning throughout life, preparing them for future jobs and society, with the same opportunities for all. This is a pillar of education, so that all individuals can access the job market and develop their abilities, and all actors in education must try to incorporate all students and reduce the abandon rates.

In addition, Cabrera [26] stated the importance of the formation in robotics for the creation of new jobs, emphasising on the low number of new graduates in computer science in Europe, the collateral advantages of programming learning (social, educative, critic reasoning, skills development, sustainability, etc.), the lack of technology and communication experts that is expected in the near future and the increase in the number of employments that need experts in digital competences. These works will require responsible individuals to ensure an ethical and sustainable development. Therefore, the teaching of programming and robotics is key in primary and secondary education. 
Among the pioneer countries in CT integration (and specially robotics) in compulsory secondary education, there is a trend to incorporate the algorithmic and programming thinking in the digital competences. These competences will be basic for the development of the new world economic order in terms of work, social relations and sustainability, in which robotics will be a key factor. Despite the fact that service robotics is becoming an emerging field, in many countries, there is still a high technology dependency in the development of robots, there is a lack of formation in small and medium companies, the investments in robotics are very limited, and there is a lack of research in robotics, among other factors [59]. This situation must be reverted, and technology development must be improved with actions that start from the teaching of these concepts in compulsory education.

Based on this evidence, and through the developed practice, we can conclude that the teaching of robotics in compulsory secondary education must be part of the standard curriculum from the early years of education. Moreover, it should be considered in the widest sense possible, motivating the students to create new solutions with a systemic approach, collaborating, and creating generalisable algorithms that could be applied to different disciplines.

Based on these ideas, in this work, the application of a teaching methodology was proposed, whose innovation was threefold: (i) the way in which the competences were evaluated; (ii) the planning of activities for each learning style; and (iii) the resources combination. The combination of the above led to a proposal for the teaching of robotics that may be applied even in the case of lack of resources and that needs a very limited investment by the institution. It is important to remark the flexibility of the proposal, which could be modified and adapted based on the learning styles and profiles of the students and on the previous experience of the teachers.

Although the results are preliminary and obtained over a small group of students, based on the qualitative research and on the results of other works with few participants [58], the outcomes obtained may be transferred to similar or different contexts. It may be difficult to carry out this transference; however, the results obtained can provide guidelines to outline a general idea of the problem studied and to apply some solutions in other contexts [54]. Those who want to use the results of an action research approach in their own contexts should determine if there are enough similarities to transfer the results [60]. Therefore, the proposal may be used as a guide for teaching CT and robotics and to promote the interest and commitment of students. The possible criticism to the weak generalisation of the action research approach may be overcome by increasing the number of qualitative studies published, so that expanding the local knowledge accumulation may enhance the transferability of the knowledge generated [61].

In addition, the aim of this proposal was to apply it to a disruptive group, with difficulties to continue the studies, with poor academic background in programming and robotics and with high degree of apathy and demotivation. The methodology was applied to the group with very promising results, and the results contrasted with the application of the same methodology to a motivated and committed group. As far as the authors know, there are no studies about the teaching of robotics and CT that started from similar student conditions.

Author Contributions: Conceptualisation, B.D.-L.; Formal analysis, D.M.-S.; Investigation, B.D.-L. and D.M.-S.; Methodology, B.D.-L. and D.M.-S.; Supervision, D.M.-S.; Validation, B.D.-L.; Writing-Original draft, B.D.-L.; and Writing-Review and editing, D.M.-S.

Funding: This work was funded by Spanish Ministry of Economy and Competitiveness under project CICYT DPI2017-84259-C2-2-R. This work was supported in part by the UNED project GID2016-6.

Conflicts of Interest: The authors declare no conflict of interest.

\section{References}

1. Kazimieras, J.; Katiliute, E. Complex evaluation of sustainability in engineering education: Case \& analysis. J. Clean. Prod. 2016, 120, 13-20. [CrossRef]

2. McLaughlan, R. Instructional Strategies to Educate in Sustainability in Technology Assessment. Int. J. Eng. Educ. 2007, 23, 201-208. 
3. UNESCO. Education for Sustainable Development Goals. Learning Objectives. Available online: http: / / unesdoc.unesco.org/images/0024/002474/247444e.pdf (accessed on 20 August 2019)

4. Berry, M. Computing in the National Curriculum. A Guide for Primary Teachers; Computing at School: Swindon, UK, 2013; ISBN 978-1-78339-143-1.

5. Tabet, N.; Gedawy, H.; Alshikhabobakr, H.; Razak, S. From Alice to Python. Introducing Text-based Programming in Middle Schools. In Proceedings of the 2016 ACM Conference on Innovation and Technology in Computer Science Education ITiCSE 2016, Arequipa, Peru, 9-13 July 2016; pp. 124-129.

6. Dwyer, H.; Hill, C.; Hansen, A.; Iveland, A.; Franklin, D.; Harlow, D. Fourth Grade Students Reading Block-Based Programs: Predictions, Visual Cues, and Affordances. In Proceedings of the Eleventh Annual International Conference on International Computing Education Research ICER 2015, Omaha, NE, USA, 9-13 August 2015; pp. 111-119.

7. Papadakis, S.; Kalogiannakis, M.; Orfanakis, V.; Zaranis, N. The Appropriateness of Scratch and App Inventor as Educational Environments for Teaching Introductory Programming in Primary and Secondary Education. Int. J. Web Based Learn. Teach. Technol. (IJWLTT) 2017, 12, 58-77. [CrossRef]

8. Papadakis, S.; Orfanakis, V. The combined use of Lego Mindstorms NXT and App Inventor for teaching novice programmers. In Proceedings of the International Conference EduRobotics 2016, Athens, Greece, 25 November 2016; pp. 193-204.

9. Papadakis, S.; Orfanakis, V. Comparing novice programming environments for use in secondary education: App Inventor for Android vs. Alice. Int. J. Technol. Enhanc. Learn. 2018, 10, 44-72. [CrossRef]

10. Blumenfeld, P.C.; Soloway, E.; Marx, R.W.; Krajcik, J.S.; Guzdial, M.; Palincsar, A. Motivating Project-Based Learning: Sustaining the Doing, Supporting the Learning. Educ. Psychol. 1991, 26, 369-398. [CrossRef]

11. Brown, A.L.; Campione, J.C. Psychological theory and the design of innovative learning environments. On procedures, principles, and systems. In Innovation in Learning: New Environments for Education; Schauble, L., Glaser, R., Eds.; Lawrence Erlbaum Associates: Hillsdale, NJ, USA, 1996; pp. 289-325, ISBN 978-0805820706.

12. Mills, J.E.; Treagust, D.F. Engineering education-Is problem-based or project-based learning the answer? Australas. J. Eng. Educ. 2003, 3, 2-16.

13. Thomas, J.W. A Review of Research on Project-Based Learning; The Autodesk: San Rafael, CA, USA, 2000.

14. Williams, M.; Grinnell, R.M.; Unrau, Y.A. Case levels design. In Social Work: Research and Evaluation. Quantitative and Qualitative Approaches, 7th ed.; Grinnell, R.M., Unrau, Y.A., Eds.; Oxford University Press: New York, NY, USA, 2005; pp. 171-184, ISBN 978-0195179491.

15. Roman-Gonzalez, M.; Pérez-González, J.C.; Moreno-León, J.; Robles, G. Extending the nomological network of computational thinking with non-cognitive factors. Comput. Hum. Behav. 2017, 80, 441-459. [CrossRef]

16. Wing, J. M. Computational thinking. Commun. ACM 2006, 49, 33-35. [CrossRef]

17. Wing, J.M. Computational thinking and thinking about computing. Philos. Trans. R. Soc. A 2008, 366, 3717-3725. [CrossRef]

18. Barr, V.; Stephenson, C. Bringing computational thinking to K-12: What is involved and what is the role of the computer science education community? Inroads 2011, 2, 48-54. [CrossRef]

19. Lee, I.; Martin, F.; Denner, J.; Coulter, B.; Allan, W.; Erickson, J.; Malyn-Smith, J.; Werner, L. Computational thinking for youth in practice. Inroads 2011, 2, 32-37. [CrossRef]

20. Grover, S.; Pea, R. Computational thinking in K-12 a review of the state of the field. Educ. Res. 2013, 42, 38-43. [CrossRef]

21. Curzon, P.; Dorling, M.; Ng, T.; Selby, C.; Woollard, J. Developing Computational Thinking in the Classroom: A Framework; Computing at School: Swindon, UK, 2014. Available online: https://eprints.soton.ac.uk/ 369594/ (accessed on 20 August 2019)

22. Ioannou, I.; Charoula A. A Framework and an Instructional Design Model for the Development of Students' Computational and Algorithmic Thinking. In Proceedings of the 10th Mediterranean Conference on Information Systems MCIS 2016, Paphos, Cyprus, 4-6 September 2016; p. 19.

23. Bocconi, S.; Chioccariello, A.; Dettori, G.; Ferrari, A.; Engelhardt, K. Developing Computational Thinking in Compulsory Education-Implications for Policy and Practice; Joint Research Centre: Seville, Spain, 2016, doi:10.2791/792158.

24. Computer Science Teacher Association. K-12 CS Standards. Available online: https://www.csteachers.org/ page/standards (accessed on 20 August 2019) 
25. Balanskat, A.; Engelhardt, K. Computing our Future. Computer Programming and Coding: Priorities, School Curricula and Initiatives Across Europe; European Schoolnet: Brussels, Belgium, 2015.

26. Cabrera, J.M. Programación informática y robótica en la enseñanza básica. Avances en Supervisión Educativa 2015, 24. [CrossRef]

27. Moreno-Leon, J; Roman-Gonzalez, M.; Robles, G. Can we Measure Computational Thinking with Tools? Present and Future of Dr. Scratch. In Proceedings of the Seminar Series on Advanced Techniques and Tools for Software Evolution SATToSE 2017, Madrid, Spain, 7-9 June 2017.

28. Google; Fundación Española para la Ciencia y la Tecnología (FECYT); Everis. Computer Science Education in Spain 2015. Available online: https://www.fecyt.es/publicacion/educacion-de-las-ciencias-de-lacomputacion-en-espana (accessed on 20 August 2019)

29. Acuña, A.; Castro, M.; Obando, D. Desarrollo de Capacidades Para el DiseñO e ImplementacióN de Proyectos de RobóTica Educativa en AméRica Latina y el Caribe; Fundación Omar Dengo: San José, Costa Rica, 2011.

30. Shoop, R.; Flot, J.; Higashi, R.; Witherspoon, E.; McKenna J. Using Model Eliciting Activities to Engage Students in Computational Thinking Practices in Robotics Classrooms. In Proceedings of the High Impact Technology Exchange Conference HI-TECH 2016, Pittsburgh, PA, USA, 25-28 July 2016.

31. Toh, D.; Ravintharan; Lim, M; Wee, L.K.; Ong, M. Robotics for Learning. arXiv 2015, arxiv:1502.01089.

32. Kanemune, S.; Shirai, S.; Seeichi, T. Informatics and programming education at primary and secondary in Japan. Olymp. Inform. 2017, 11, 143-150. [CrossRef]

33. Japan Info. A Drone and Robotics Course for High School Students? Only in Japan! Available online: https://jpninfo.com/63059 (accessed on 20 August 2019)

34. Liu, X.; Gong, X.; Wang, F.; Sun, R.; Gao, Y.; Zhang, Y.; Zhou, J.; Deng, X. A New Framework of Science and Technology Innovation Education for K-12 in Qingdao. In Proceedings of the 2017 American Society for Engineering Education International Forum ASEE 2017, Columbus, OH, USA, 28 June 2017.

35. Bong-Hyun, K. Development of young children coding drone using block game. Indian J. Sci. Technol. 2016, 9, 1-5. [CrossRef]

36. Mikropoulos, T. A.; Bellou, I. Educational robotics as mindtools. Themes Sci. Technol. Educ. 2013, 6, 5-14.

37. Perez, R.; Galan, A.; Quintanal, J. Research Methods and Designs in Education; Universidad de Educación a Distancia (UNED): Madrid, Spain, 2012; ISBN: 9788436262667.

38. Sandín, M. Qualitative Research in Education. Fundamentals and Traditions; McGrawHill: New York, NY, USA, 2003; ISBN 9788448137793.

39. Cerdán R.; Gil, L. Cognitive development and learning processes. In Personality Learning and Development; Vidal Abarca, E., Garcia, R., Pérez, F., Eds.; Alianza Editorial: Madrid, Spain, 2010; pp. 45-70, ISBN 978-84-206-8908-1.

40. Polman, J.L. The zone of proximal identity development in apprenticeship learning. Rev. Educ. 2010, 353, 129-155.

41. Ausubel, D.P. AdquisicióN y RetencióN del Conocimiento: Una Perspectiva Cognitiva; Paidós Ibérica: Barcelona, Spain, 2002; ISBN 978-84-493-1234-2.

42. Papert, S. Hard Fun; Bangor Daily News: Bangor, Maine, 2011.

43. Bruner, J.S. The Process of Education; Harvard University Press: Oxford, UK, 1960; ISBN 0674710010.

44. Csikszentmihalyi, M. Flow and the Foundations of Positive Psychology; Springer: Berlin, Germany, 2014; ISBN 978-94-017-9088-8.

45. Chaplin, J.P.; Krawiec, T.S. Systems and Theories of Psychology, 4th ed.; Holt, Rinehart and Winston: New York, NY, USA, 1979; ISBN9780030202711.

46. Robinson, K. Finding Your Element; DK: London, UK, 2014; ISBN 9780241952023.

47. Tello-Leal, E.; Guerrero-Melendez, T.; Saldívar-Alonso, V. A review of Lego Mindstorms robotics platform for educational and research applications. Sist. Telemát. 2013, 11, 9-27. [CrossRef]

48. Blockly Games. Available online: https://blockly-games.appspot.com/ (accessed on 20 August 2019).

49. Sabandar, N.; Noldy, R.; Suryana, E. Kahoot!: Bring the Fun Into the Classroom!. Indones. J. Inform. Educ. 2018, 2, 127-134. [CrossRef]

50. Selltiz, C.; Jahoda, M.; Deutsch, M.; Cook, S.W. Research Methods in Social Relations; Holt, Rinehart and Winston: New York, NY, USA, 1959; ISBN 978-0030311499.

51. Atlas.ti: Qualitative Data Analysis. Available online: https:/ /atlasti.com/ (accessed on 20 August 2019) 
52. Weitzman, E.; Miles, M. Computer Programs for Qualitative Data Analysis; SAGE Publications: London, UK, 1995; ISBN 0-8039-5537-5.

53. Lewins, A.; Silver, C. Using Software in Qualitative Research: A Step-by-Step Guide; SAGE Publications: London, UK, 2014; ISBN 9781446249734.

54. Sampieri, H. Methodology of the Research; McGrawHill: New York, NY, USA, 2014; ISBN: 978-1-4562-2396-0.

55. Mertens, D. Research and Evaluation in Education and Psychology: Integrating Diversity with Quantitative, Qualitative, and Mixed Methods; SAGE Publications: London, UK, 2010; ISBN 978-1-4129-7190-4.

56. Savin-Baden, M.; Major, C.H. Qualitative Research: The Essential Guide to Theory and Practice; Routledge: Abingdon, UK, 2013; ISBN 978-0-415-67478-2.

57. Grover, S.; Bienkowski, M.; Niekrasz, J.; Hauswirth, M. Assessing Problem-Solving Process At Scale. In Proceedings of the Third ACM Conference on Learning At Scale 2016, Edinburgh, UK, 25-26 April 2016.

58. Nilsson, L.; Eklund, M.; Nyberg, P.; Thulesius, H. Driving to learn in a powered wheelchair: The process of learning joystick use in people with profound cognitive disabilities. Am. J. Occup. Ther. 2011, 65, 652-660. [CrossRef] [PubMed]

59. Comité Español de Automática. The White Book of Robotics in Spain. Research, Technologies and Training; CEA-GTRob: Madrid, Spain, 2011; ISBN 978-84-615-4583-4

60. Somekh, B. Action Research: A Methodology for Change and Development; McGraw-Hill Education: Berkshire, UK, 2006; ISBN 0-3352-1659-5.

61. Brabury-Huang, H. What is good action research? Why resurgent interest? Act. Res. 2010, 8, 93-109. [CrossRef]

(C) 2019 by the authors. Licensee MDPI, Basel, Switzerland. This article is an open access article distributed under the terms and conditions of the Creative Commons Attribution (CC BY) license (http://creativecommons.org/licenses/by/4.0/). 